\title{
Optimization of Open Boundary Conditions in a 3D Internal Tidal Model with the Adjoint Method around Hawaii
}

\author{
Anzhou Cao, ${ }^{1}$ Haibo Chen, ${ }^{1}$ Jicai Zhang, ${ }^{2}$ and Xianqing Lv ${ }^{1}$ \\ ${ }^{1}$ Laboratory of Physical Oceanography, Ocean University of China, Qingdao 266003, China \\ ${ }^{2}$ Laboratory of Coast and Island Development, Nanjing University, Nanjing 210093, China \\ Correspondence should be addressed to Xianqing Lv; xqinglv@ouc.edu.cn
}

Received 4 January 2013; Accepted 13 March 2013

Academic Editor: Guanglu Zhou

Copyright (c) 2013 Anzhou Cao et al. This is an open access article distributed under the Creative Commons Attribution License, which permits unrestricted use, distribution, and reproduction in any medium, provided the original work is properly cited.

\begin{abstract}
Based on the theory of inverse problem, the optimization of open boundary conditions (OBCs) in a 3D internal tidal model is investigated with the adjoint method. Fourier coefficients of $M_{2}$ internal tide on four open boundaries, which are regarded as OBCs, are inverted simultaneously. During the optimization, the steepest descent method is used to minimize cost function. The reasonability and feasibility of the model are tested by twin experiments (TEs). In TE1, OBCs on four open boundaries are successfully inverted by using independent point (IP) strategy, suggesting that IP strategy is useful in parameter estimation. Results of TE2 indicate that the model is effective even by assimilating inaccurate "observations." Based on conclusions of TEs, the $M_{2}$ internal tide around Hawaii is simulated by assimilating T/P data in practical experiment. The simulated cochart shows good agreement with that obtained from the Oregon State University tidal model and T/P observations. Careful inspection shows that the major difference between simulated results and OSU model results is short-scale fluctuations superposed on coamplitude lines, which can be treated as the surface manifestation modulated by the internal tide. The computed surface manifestation along $\mathrm{T} / \mathrm{P}$ tracks is comparable to the estimation in previous work.
\end{abstract}

\section{Introduction}

Internal tide is an important intermediate step of the tidesto-turbulence cascade [1] and plays an important role in the dissipation of surface tides and mixing in the deep ocean so as to have strong influence on the global thermohaline circulation [2]. The Hawaiian Ridge is one of the most suitable areas to investigate the internal tides. Rudnick et al. [1], Ray and Mitchum [3, 4], Munk [5], Martin et al. [6], and Zhao et al. [7-9] investigated internal tide along the Hawaiian Ridge by using data of T/P satellite altimetry, Hawaii Ocean Mixing Experiment (HOME), and many other observations such as acoustics Doppler current profilers (ADCPs). Kang et al. [10], Simmons et al. [11], Carter et al. [12], Powell et al. [13], Rainville et al. [14], and Zaron and Egbert [15] used numerical models to simulate internal tides and study the mechanism of internal tide. Through their work, the generation, propagation, vertical structure, energy budget, and other characteristics of internal tide around Hawaii have been described preliminarily.
Among all data assimilation methods, the $4 \mathrm{D}$ variational data assimilation is one of the most effective approaches and widely used in meteorological and oceanographic models [16-32]. Based on the theory of inverse problem, it is an advanced data assimilation method which involves the adjoint method and has the advantage of assimilating various observations disturbed in time and space [22-37]. Lardner [22], Myers and Baptista [23], Zhang and Lu [24], Guo et al. [25], and Cao et al. [26] studied the inverse problem of OBCs in barotropic tidal models. Chen et al.s [27] constructed a 3D isopycnic-coordinate internal tidal model with adjoint method and studied inverse problem of OBCs at 14 kinds of bottom topographies. As for the inverse problem of other parameters, Lu and Zhang [28] and Zhang et al. [29] inverted spatially varying bottom friction coefficient in the 2D tidal model and Yu and O'Brien [30] estimated wind drag coefficient and eddy viscosity in the Ekman layer model.

The work of this paper is an extension of Chen et al. [27], in which Fourier coefficients of $M_{2}$ internal tide on four open boundaries are inverted simultaneously. During 
the optimization, steepest descent method with special step factor is used to minimize cost function. This paper is organized as follows. The 3D internal tidal model with adjoint method, minimization algorithm, independent point (IP) strategy and model settings are introduced in Section 2. Identical twin experiments (TEs) are carried out to test the model in Section 3. Practical experiment (PE) and its analysis are given in Section 4. Finally, the summary and conclusions are provided in Section 5.

\section{Numerical Model and Settings}

2.1. Forward Model. The governing equations of internal tide in spherical coordinate are described as follows:

$$
\begin{aligned}
\frac{\partial q_{k}}{\partial t} & +\frac{1}{a} \frac{\partial\left(q_{k} u_{k}\right)}{\partial \lambda}+\frac{1}{a} \frac{\partial\left(q_{k} v_{k} \cos \varphi\right)}{\partial \varphi}=0 \\
\frac{\partial u_{k}}{\partial t}+ & L\left(u_{k}\right)-f v_{k} \\
& =-\frac{1}{a} \frac{\partial M_{k}}{\partial \lambda}-\frac{g}{a} \frac{\partial \bar{\zeta}}{\partial \lambda}+\frac{\tau_{(k-1) x}-\tau_{k x}}{q_{1}}+A_{h} \Delta u_{k} \\
\frac{\partial v_{k}}{\partial t}+ & L\left(v_{k}\right)+f u_{k} \\
& =-\frac{1}{r} \frac{\partial M_{k}}{\partial \varphi}-\frac{g}{r} \frac{\partial \bar{\zeta}}{\partial \varphi}+\frac{\tau_{(k-1) y}-\tau_{k y}}{q_{1}}+A_{h} \Delta v_{k}
\end{aligned}
$$

in which,

$$
\begin{gathered}
L\left(u_{k}\right)=\frac{u_{k}}{a} \frac{\partial u_{k}}{\partial \lambda}+\frac{v_{k}}{r} \frac{\partial u_{k}}{\partial \varphi}-\frac{u_{k} v_{k} \tan \varphi}{r}, \\
L\left(v_{k}\right)=\frac{u_{k}}{a} \frac{\partial v_{k}}{\partial \lambda}+\frac{v_{k}}{r} \frac{\partial v_{k}}{\partial \varphi}+\frac{u_{k}^{2} \tan \varphi}{r}, \\
\Delta u_{k}=\frac{1}{a^{2}} \frac{\partial^{2} u_{k}}{\partial \lambda^{2}}+\frac{1}{r a} \frac{\partial}{\partial \varphi}\left(\frac{\partial u_{k}}{\partial \varphi} \cos \varphi\right), \\
\Delta v_{k}=\frac{1}{a^{2}} \frac{\partial^{2} v_{k}}{\partial \lambda^{2}}+\frac{1}{r a} \frac{\partial}{\partial \varphi}\left(\frac{\partial v_{k}}{\partial \varphi} \cos \varphi\right), \\
M_{k}=g\left(\frac{q}{\rho}-D\right)+g \sum_{m=1}^{k-1}\left[\left(\frac{1}{\rho}-\frac{1}{\rho_{k}}\right) q_{m}\right]
\end{gathered}
$$

where $k=1,2, \ldots, N$ is vertical layer index, $t$ is time, $\lambda$ and $\varphi$ are longitude and latitude, $q_{k}, u_{k}$ and $v_{k}$ are water mass and horizontal velocity of the $k$ th layer, respectively, $f$ is the Coriolis parameter, $\bar{\zeta}$ is the tidal potential, $A_{h}$ is the horizontal viscosity coefficient, $r$ is the radius of the earth, $a=r \cos \varphi, g$ is the gravity, $D$ is the total depth at rest, $q=\sum_{k=1}^{N} q_{k}$ is the total mass of the water column, $\rho=$ $q / \sum_{k=1}^{N}\left(q_{k} / \rho_{k}\right)$ is the mean density, $\tau_{0 x}$ and $\tau_{0 y}$ are wind stress components (in the paper, they are equal to zero), $\tau_{N x}$ and $\tau_{N y}$ are bottom friction components $\left(\tau_{N x}=\kappa \rho_{N} u_{N} \sqrt{u_{N}^{2}+v_{N}^{2}}\right.$, $\tau_{N y}=\kappa \rho_{N} v_{N} \sqrt{u_{N}^{2}+v_{N}^{2}}$ in which $\kappa$ is the nondimensional bottom friction coefficient), and $\tau_{k x}$ and $\tau_{k y}(k=1, \ldots, N-1)$ are interfacial friction between the $k$ th layer and the $(k+1)$ th layer, and they are defined as

$$
\begin{gathered}
\tau_{k x}=\mu_{k}\left(u_{k}-u_{k+1}\right), \quad \tau_{k y}=\mu_{k}\left(v_{k}-v_{k+1}\right), \\
\mu_{k}=A_{v} \frac{\rho_{k}+\rho_{k+1}}{h_{k}+h_{k+1}},
\end{gathered}
$$

where $A_{v}$ is the vertical viscosity coefficient; $h_{k}$ is the undisturbed thickness of the $k$ th layer.

Equations (1) and its vertical integration, which are called the internal and external mode, respectively, compose the forward model and are used to simulate the internal tide. Miao et al. [31] used this model to investigate the characteristic of generation and propagation of $M_{2}$ and $K_{1}$ internal tides in the South China Sea.

The Flather condition [38] can yield good results [27, 31] when applied to the open boundaries in this model. In this paper, an adaptation of the Flather condition is installed in the external mode of the forward model, which is given as follows:

$$
\zeta=\widetilde{\zeta} \pm \sqrt{\left(1-\frac{f^{2}}{\omega^{2}}\right) \frac{H}{g}} U, \quad \zeta=\widetilde{\zeta} \pm \sqrt{\left(1-\frac{f^{2}}{\omega^{2}}\right) \frac{H}{g}} V
$$

where $\widetilde{\zeta}$ is external data beyond the model boundary representing the clamped surface elevation relating to the boundary barotropic tidal force, $H$ is the time-varying total water depth, and $U$ and $V$ are barotropic velocity. The signs in (5) are positive for eastern and northern boundaries and negative for western and southern boundaries. Equations (5) can be determined once the external data $\widetilde{\zeta}$ is described. The $M_{2}$ tidal force $\widetilde{\zeta}$ at the $n$th time step along the open boundary can be described as

$$
\widetilde{\zeta}_{i i, j j}^{n}=a_{i i, j j} \cos (\omega n \Delta t)+b_{i i, j j} \sin (\omega n \Delta t),
$$

where $a$ and $b$ are Fourier coefficients of OBCs, $\omega$ is the frequency of $M_{2}$ tide, and $\Delta t$ is time step. So the inverse problem of OBCs is turned into the inverse problem of Fourier coefficients $a$ and $b$.

For the internal mode, the relaxation conditions are applied. A relaxation region of several grids close to the boundary is defined. In this region, tidal values including the interface elevation and fluid velocity of both barotropic and baroclinic tides are first computed and then relaxed to final values displayed as follow

$$
x(i, j)=x_{B t}(i, j)+[1-\gamma(i, j)] \cdot x_{B c}(i, j),
$$

where $x$ represents the tidal state $\eta_{k}, u_{k}$, or $v_{k}, x_{B t}$ and $x_{B c}$ represent the barotropic and baroclinic tides, respectively, and $\gamma(i, j) \in[0,1]$ is the relaxation factor in grid $(i, j)$. In our model, $\gamma$ is increased from 0 to 1 as the grid is getting close to the open boundary. 
In addition, the initial conditions are that the initial surface elevation, interfacial elevation, $x$ - and $y$-velocity at all layers are zeros.

2.2. Adjoint Model. The adjoint model is similar to that in Chen et al. [27], which can be used to optimize OBCs and/or other spatially varying parameters and displayed as follows.

The cost function is defined by

$$
J(q, u, v ; \mathbf{p})=\frac{1}{2} \int_{\Sigma}\left(\frac{q}{\rho}-D-\widehat{\zeta}\right)^{2} d \sigma
$$

where $\Sigma$ is the model domain of both time and space, $\widehat{\zeta}$ is the observations, and $\mathbf{p}$ represents the control variables and is defined by the OBCs, which are denoted by a series of Fourier coefficients in this work.

The Lagrangian function is defined by

$$
\begin{aligned}
& L\left(q, u, v, q_{a}, u_{a}, v_{a} ; \mathbf{p}\right) \\
& =J(q, u, v ; \mathbf{p})+\int_{\Sigma}\left[q _ { a 1 } \left(\frac{\partial q_{1}}{\partial t}+\frac{1}{a} \frac{\partial\left(q_{1} u_{1}\right)}{\partial \lambda}\right.\right. \\
& \left.\left.+\frac{1}{a} \frac{\partial\left(q_{1} v_{1} \cos \varphi\right)}{\partial \varphi}\right)\right] d \sigma \\
& +\int_{\Sigma}\left[u _ { a 1 } \left(\frac{\partial u_{1}}{\partial t}+L\left(u_{1}\right)-f v_{1}+\frac{1}{a} \frac{\partial M_{1}}{\partial \lambda}\right.\right. \\
& \left.\left.+\frac{g}{a} \frac{\partial \bar{\zeta}}{\partial \lambda}-\frac{\tau_{0 x}-\tau_{1 x}}{q_{1}}-A_{h} \Delta u_{1}\right)\right] d \sigma \\
& +\int_{\Sigma}\left[v _ { a 1 } \left(\frac{\partial v_{1}}{\partial t}+L\left(v_{1}\right)+f u_{1}+\frac{1}{r} \frac{\partial M_{1}}{\partial \varphi}\right.\right. \\
& \left.\left.+\frac{g}{r} \frac{\partial \bar{\zeta}}{\partial \varphi}-\frac{\tau_{0 y}-\tau_{1 y}}{q_{1}}-A_{h} \Delta v_{1}\right)\right] d \sigma \\
& +\cdots \\
& +\int_{\Sigma}\left[q_{a N}\left(\frac{\partial q_{N}}{\partial t}+\frac{1}{a} \frac{\partial\left(q_{N} u_{N}\right)}{\partial \lambda}+\frac{1}{a} \frac{\partial\left(q_{N} v_{N} \cos \varphi\right)}{\partial \varphi}\right)\right] d \sigma \\
& +\int_{\Sigma}\left[u _ { a N } \left(\frac{\partial u_{N}}{\partial t}+L\left(u_{N}\right)-f v_{N}+\frac{1}{a} \frac{\partial M_{N}}{\partial \lambda}\right.\right. \\
& \left.\left.+\frac{g}{a} \frac{\partial \bar{\zeta}}{\partial \lambda}-\frac{\tau_{(N-1) x}-\tau_{N x}}{q_{1}}-A_{h} \Delta u_{N}\right)\right] d \sigma \\
& +\int_{\Sigma}\left[v _ { a N } \left(\frac{\partial v_{N}}{\partial t}+L\left(v_{N}\right)+f u_{N}+\frac{1}{r} \frac{\partial M_{N}}{\partial \varphi}\right.\right. \\
& \left.\left.+\frac{g}{r} \frac{\partial \bar{\zeta}}{\partial \varphi}-\frac{\tau_{(N-1) y}-\tau_{N y}}{q_{1}}-A_{h} \Delta v_{N}\right)\right] d \sigma,
\end{aligned}
$$

where $q_{a k}, u_{a k}$ and $v_{a k}$ are adjoint variables of $q_{k}, u_{k}$, and $v_{k}$, respectively.
According to the typical theory of Lagrangian multiplier method, we have the following first-order derivates of Lagrangian function with respect to all the variables and parameters:

$$
\begin{aligned}
& \frac{\partial L}{\partial q_{a k}}=0, \quad \frac{\partial L}{\partial u_{a k}}=0, \quad \frac{\partial L}{\partial v_{a k}}=0, \\
& \frac{\partial L}{\partial q_{k}}=0, \quad \frac{\partial L}{\partial u_{k}}=0, \quad \frac{\partial L}{\partial v_{k}}=0, \\
& \frac{\partial L}{\partial \mathbf{p}}=0 .
\end{aligned}
$$

Equations (10) are (1) actually. The adjoint equations can be derived from (11). From (12), the gradients of the cost function with respect to control variables can be obtained and displayed as follows:

$$
\begin{gathered}
\frac{\partial J}{\partial a_{i i, j j}}+\sum_{n} T_{i i, j j}^{n} \cos (\omega n \Delta t)=0, \\
\frac{\partial J}{\partial b_{i i, j j}}+\sum_{n} T_{i i, j j}^{n} \sin (\omega n \Delta t)=0 .
\end{gathered}
$$

Details on computing $T_{i i, j j}^{n}$ are given in [27].

2.3. Minimization Algorithm. Considering that the aim is to make the simulated results closer to the observations, there is the optimization problem as follows:

$$
\min : J(x) \text {, }
$$

where $J$ is the cost function; $x$ represents Fourier coefficients $a$ and $b$. Suppose that the dimension of $x$ is $N$ and the gradient of $J$ is $\nabla J\left(x_{i}^{k}\right)$ with respect to the $i$ th parameter $(1 \leq i \leq N)$ and the $k$ th iteration step. According to the typical steepest descent method,

$$
x_{i}^{k+1}=x_{i}^{k}+\alpha^{k} \cdot p_{i}^{k}
$$

where $p_{i}^{k}$ is the negative of $\nabla J\left(x_{i}^{k}\right) ; \alpha^{k}$ is the step factor at the $k$ th iteration step. The initial parameter $x_{i}^{0}$ should be prescribed (in the following experiments, $x_{i}^{0}=0$ ). In order to minimize $J$, the step factor $\alpha^{k}$ used in this paper is performed as follows:

$$
\alpha^{k}=\frac{\alpha_{0}}{\left\|\nabla J\left(x_{i}^{1}\right)\right\|_{2}}
$$

where $\alpha_{0}$ is a constant which can be obtained by prior experience; $\left\|\nabla J\left(x_{i}^{1}\right)\right\|_{2}$ is the $L_{2}$ norm of gradient at the first iteration step. Then formula (6) is changed into

$$
\begin{aligned}
& x_{i}^{k+1}=x_{i}^{k}-\alpha_{0} \cdot \frac{\nabla J\left(x_{i}^{k}\right)}{\left\|\nabla J\left(x_{i}^{1}\right)\right\|_{2}} \\
& \text { or } \quad x_{i}^{k+1}=x_{i}^{k}-\alpha_{0} \cdot \frac{\left\|\nabla J\left(x_{i}^{k}\right)\right\|_{2}}{\left\|\nabla J\left(x_{i}^{1}\right)\right\|_{2}} \cdot \frac{\nabla J\left(x_{i}^{k}\right)}{\left\|\nabla J\left(x_{i}^{k}\right)\right\|_{2}},
\end{aligned}
$$




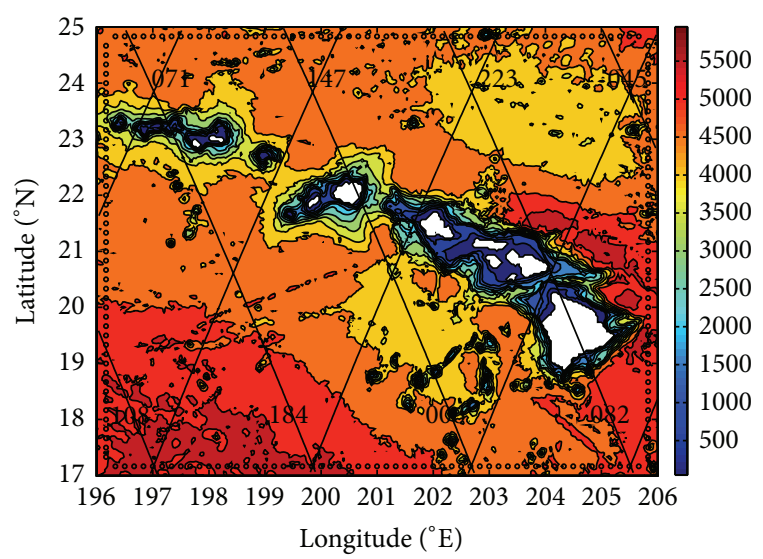

FIgURE 1: Bathymetry and T/P tracks around Hawaii ("o" represents grid point on the open boundary).

where $\nabla J\left(x_{i}^{k}\right) /\left\|\nabla J\left(x_{i}^{k}\right)\right\|_{2}$ is the normalized gradient at the $k$ th iteration step and $\left\|\nabla J\left(x_{i}^{k}\right)\right\|_{2} /\left\|\nabla J\left(x_{i}^{1}\right)\right\|_{2}$ is the ratio between $L_{2}$ norm of gradient at the $k$ th iteration step and that at the first iteration step, which could be a decreasing value as iteration step is increased.

2.4. Independent Point Strategy. Independent point strategy is considered to be a useful tool in the estimation of OBCs and spatially varying parameters. On one hand, adopting IP strategy could decrease the the number of variables so as to increase the well posedness of inverse problem. It is generally accepted that number of observations should be larger than that of variables and if number of observations is almost equal to or smaller than that of variables, the inverse problem will become ill-posed and the inverse results may be bad. On the other hand, adopting IP strategy could ensure the continuity of variables, which accords with the physical properties of variables. Lu and Zhang [28] studied the inversion problem of spatially varying bottom frication coefficients by using adjoint method and IP stragety. Results indicated that adopting IP strategy in which IPs are distributed according to depth could lead to a better simulation. Based on the theory of inverse problem, Zhang and Lu [24] simulated 3D tidal current in the Bohai and Yellow Sea by assimilating the satellite altimetry. They found that too large number of variables would lead to inaccurate inversion results, while too small number of IPs could not figure out the real condition of ocean. Guo et al. [25] and Cao et al. [26] investigated different IP strategies in a 2D tidal adjoint model. They tested the sensitivity of the model to the IP distribution, interpolation radius, and relation between prescribed $\mathrm{OBCs}$ and IP strategies.

IP strategy can be described as follows. It is assumed that several IPs exist along the open boundary (corresponding to the inversion of OBCs) or in the domain area (corresponding to the inversion of spatially varying parameters). Values at the IPs are optimized with adjoint method and those at other points are determined by linearly interpolating the values at IPs.

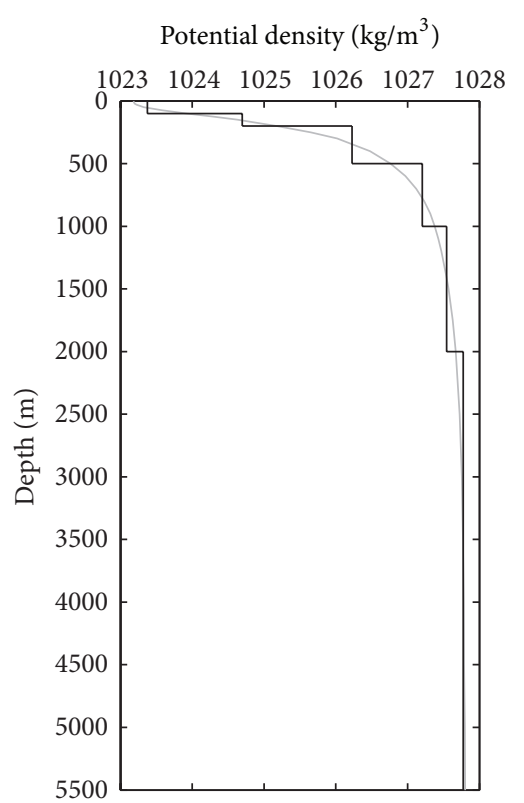

(a)

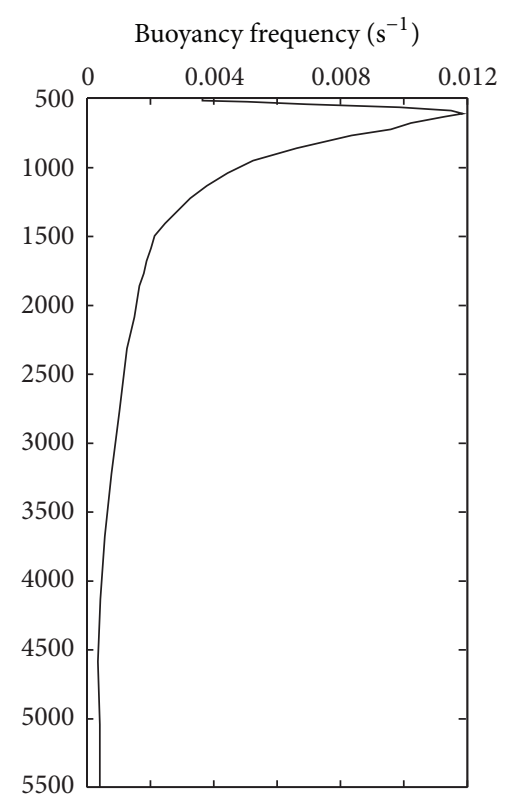

(b)

FIGURE 2: Area-averaged potential density and buoyancy frequency.

2.5. Settings. The area of interest is around Hawaii, from $196^{\circ} \mathrm{E}$ to $206^{\circ} \mathrm{E}$ and from $17^{\circ} \mathrm{N}$ to $25^{\circ} \mathrm{N}$. Figure 1 shows the bathymetry and $\mathrm{T} / \mathrm{P}$ tracks in this area. In this model, horizontal resolution is $10^{\prime} \times 10^{\prime}$ and time step is one sixtieth of the $M_{2}$ tidal period. According to area-averaged potential density and buoyancy frequency calculated from temperature and salinity data of World Ocean Atlas 2005 (WOA05), six uneven vertical layers are set varying from $100 \mathrm{~m}$ at the top to more than $4000 \mathrm{~m}$ at the bottom (shown in Figure 2). The initial density is set to be horizontally homogeneous. The non-dimensional bottom friction coefficient $\kappa$, horizontal 

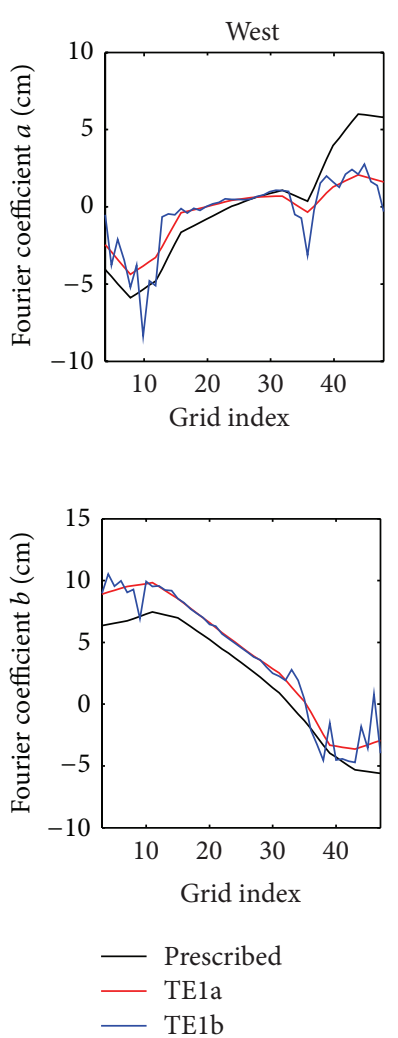

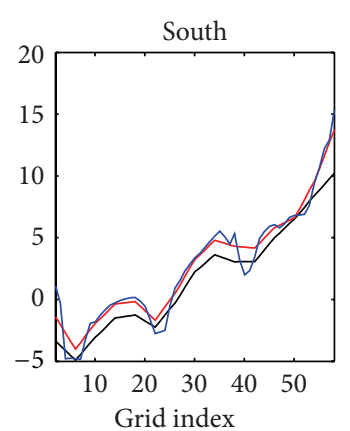

(a)

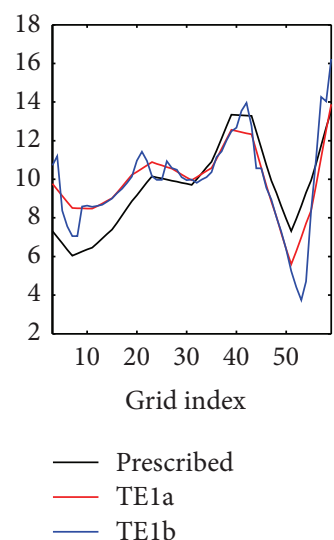

(b)
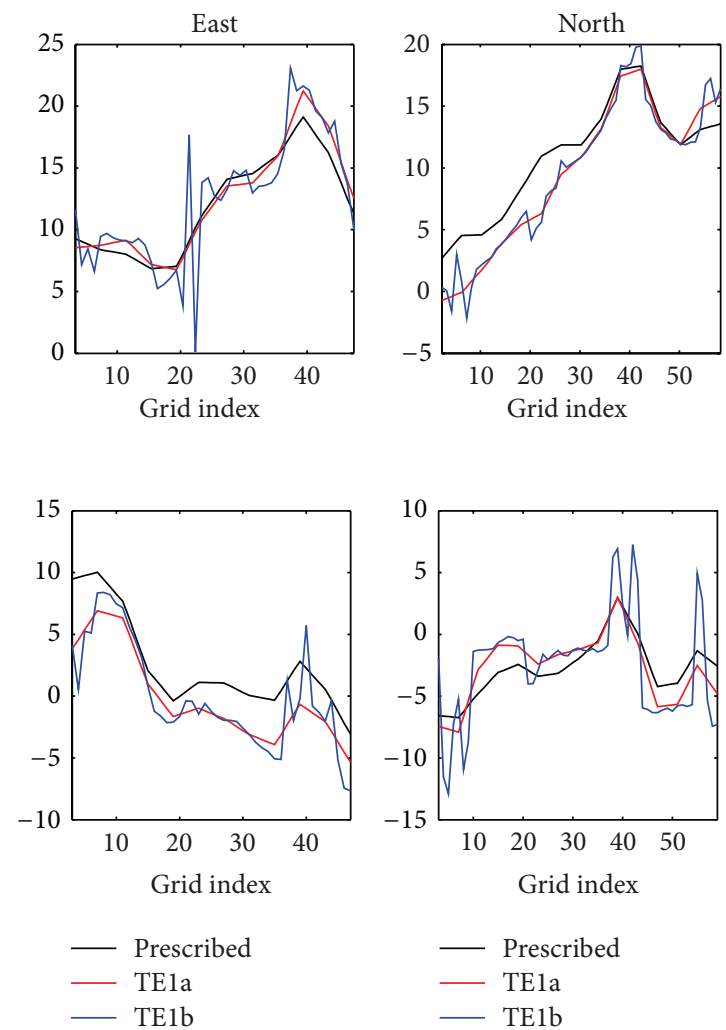

FIgUre 3: Prescribed OBCs and inverted ones in TE1.

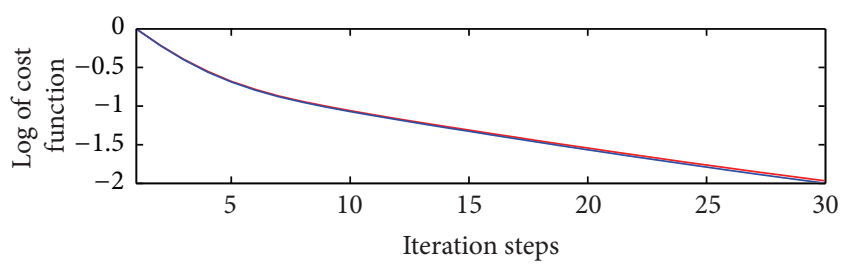

(a)

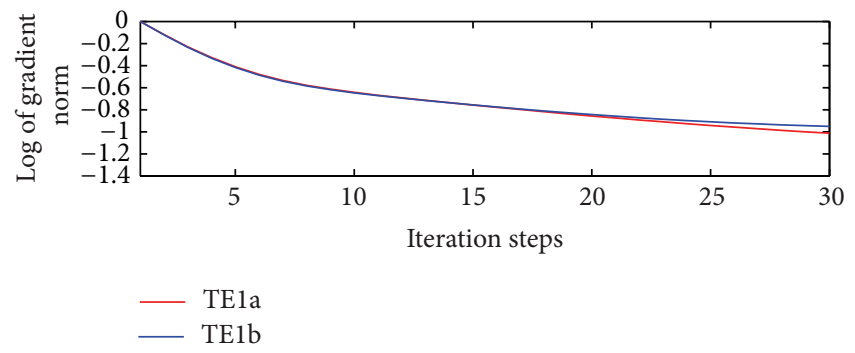

(b)

FIgURE 4: Cost function and gradient norm in TE1.

eddy viscosity $A_{h}$, and vertical eddy viscosity $A_{v}$ are 0.003 , $1000 \mathrm{~m}^{2} / \mathrm{s}$, and $0.005 \mathrm{~m}^{2} / \mathrm{s}$, respectively.

\section{Twin Experiments}

TEs are designed to test the reasonability and feasibility of the model. The results and conclusions of TEs are useful for PE. The process of TE is designed as follow. (a) Run the forward model with prescribed OBCs. The simulated sea surface elevation recorded at the grid points on T/P satellite tracks is taken as the "observations." Here only the positions of T/P tracks are used and the "observations" are not the real observations but the simulated results of forward model based on the prescribed OBCs. (b) Initial values of Fourier coefficients (taken as 0 here) are assigned to the OBCs to run the forward model. The values of state variables are obtained. (c) The difference of sea surface elevation between simulated results and "observations" serves as the external force of the adjoint model. The adjoint variables are obtained through the backward integration of the adjoint equations. (d) After obtaining the values of state variables and adjoint variables, the gradient of Fourier coefficients can be computed and the Fourier coefficients can be updated. Repeat (b), (c), and (d); the Fourier coefficients will be optimized and the difference of sea surface elevation between simulated results and "observations" will decrease. Once some convergence criterion is met, the process of inversion terminates.

The iteration will be terminated once a convergence criterion is met. The criterion could be that the last two values 

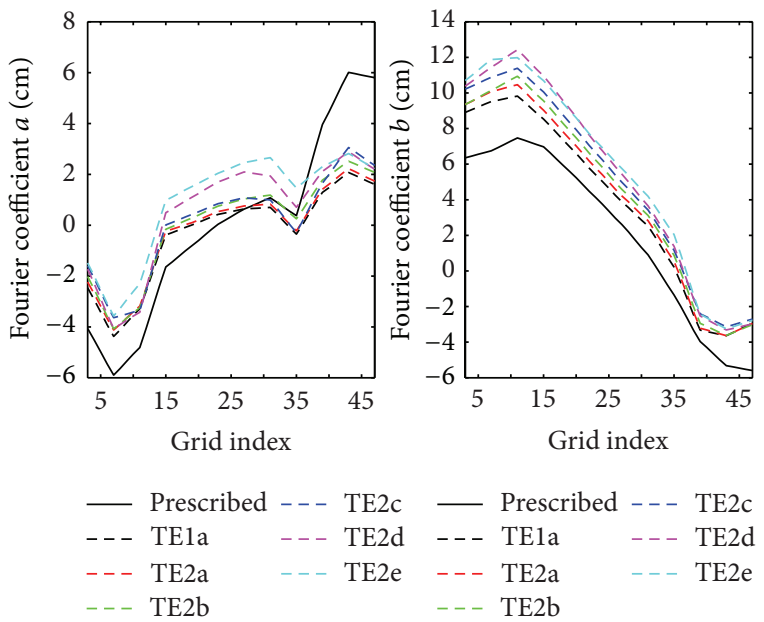

(a)
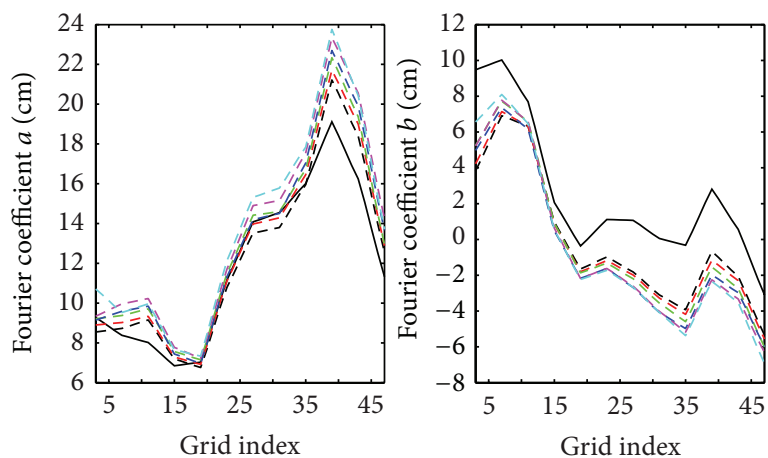

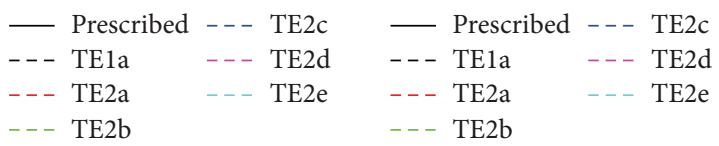

(c)
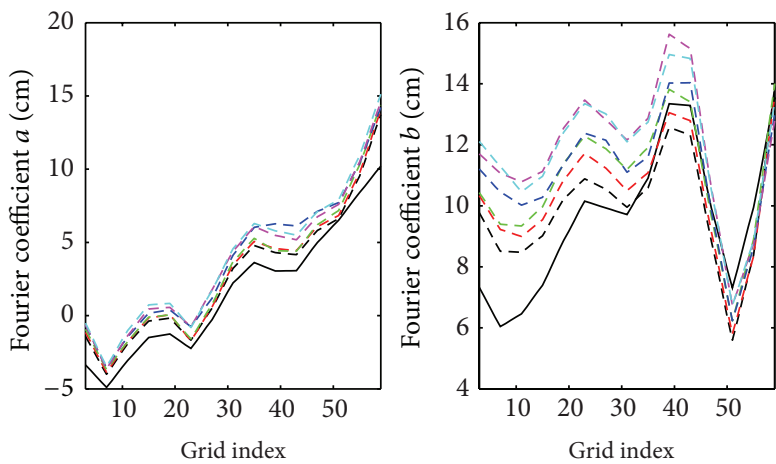

— Prescribed - - TE2c

- _. TE1a _ - TE2d

-. TE2a $\mathrm{a} \ldots$ TE2e

- - TE2b

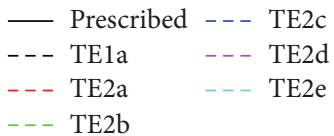

(b)
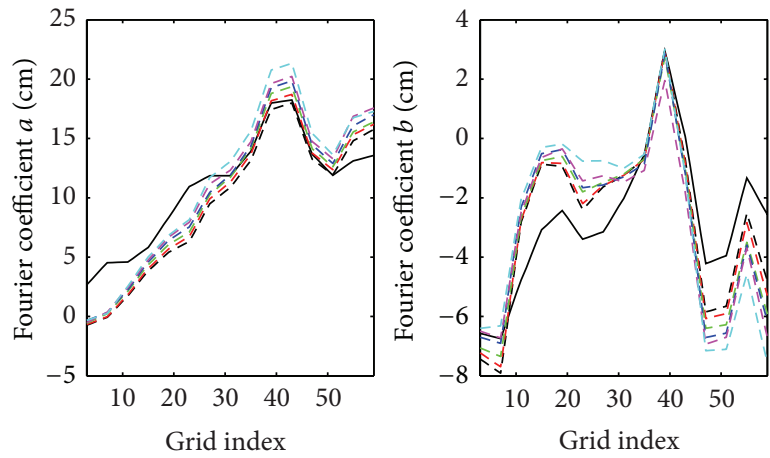

— Prescribed - - TE2c

- - - TE1a - - TE2d

- - TE2a

- - TE2b

$\begin{array}{lll}\text { - Prescribed } & --- \text { TE2c } \\ --- \text { TE1a } & --- \text { TE2d } \\ --- \text { TE2a } & --- \text { TE2e } \\ --- \text { TE2b } & \end{array}$

(d)

Figure 5: (a) Prescribed OBCs and inverted ones on the west open boundary in TE2. (b) Prescribed OBCs and inverted ones on the south open boundary in TE2. (c) Prescribed OBCs and inverted ones on the east open boundary in TE2. (d) Prescribed OBCs and inverted ones on the north open boundary in TE2.

of the cost function are sufficiently close, the magnitude of the gradient is sufficiently small, the discrepancy between the updated and old parameters is sufficiently small, or a combination of these [32]. In our work, the criterion of TEs is that the number of iteration steps is equal to 30 exactly.

\subsection{Inversion of Fourier Coefficients on Four Open Boundaries.} In this section, we study the inverse problem of Fourier coefficients on four open boundaries simultaneously, which means more variables are estimated compared with the work of Chen et al. [27] where only Fourier coefficient a on west open boundary is inverted. In addition, the feasibility and efficiency of IP strategy are tested in this section.

Figure 3 is the snapshot of prescribed OBCs and the inverse values. In TEla, IP strategy in which IPs are distributed evenly every four grid points on all four open boundaries is employed, while in TE1b no IP strategy is used.
As a whole, the shapes of inverted OBCs are similar to those of prescribed ones in both TEla and TElb. But it cannot be denied that inverted OBCs are disordered with fluctuations in TE1b where no IP strategy is used, implying the ill-posedness of inverse problem in TE1b and revealing feasibility and efficiency of IP strategy. Figure 4 shows the cost function and gradient norm which are normalized by their initial values at the first iteration step. As iteration step increased, the values of cost function and gradient norm are decreased obviously and at the end of iteration could reach $1 \%$ and $10 \%$ of their initial values, respectively, implying the optimizing ability of the model. In addition, as we use the step factor mentioned in Section 2, the cost function decreases efficiently without fluctuations. Tables 1 and 2 report the mean absolute errors (MAEs) and relative coefficients (RCs) between inverted OBCs and prescribed ones in TEla and TElb, respectively. All MAEs and RCs are less than $3 \mathrm{~cm}$ and greater than 0.7 , respectively, for both Fourier coefficients $a$ and $b$ and on every 


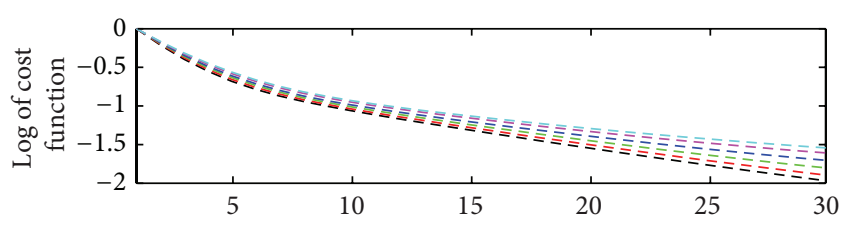

(a)

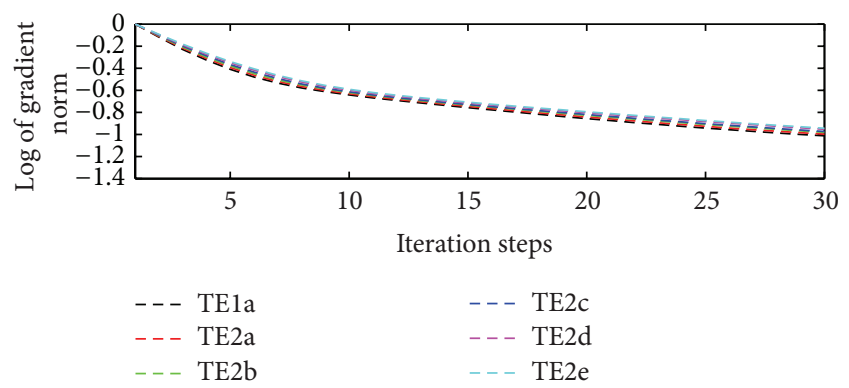

(b)

FIgURE 6: Cost function and gradient norm in TE2.

TABLE 1: MAEs between inverted OBCs and prescribed ones in TE1 (unit: $\mathrm{cm}$ ).

\begin{tabular}{lccccc}
\hline & \multicolumn{2}{c}{ TE1a } & \multicolumn{3}{c}{ TE1b } \\
& $a$ & $b$ & $a$ & $b$ \\
\hline West & 1.5 & 1.7 & 1.7 & 1.8 \\
South & 1.1 & 1.2 & 1.3 & 1.4 \\
East & 0.8 & 2.6 & 2.0 & 2.7 \\
North & 1.9 & 1.2 & 2.0 & 2.6 \\
\hline
\end{tabular}

TABLE 2: RCs between inverted OBCs and prescribed ones in TE1.

\begin{tabular}{lccccc}
\hline & \multicolumn{3}{c}{ TEla } & \multicolumn{2}{c}{ TE1b } \\
& $a$ & $b$ & $a$ & $b$ \\
\hline West & 0.94 & 0.99 & 0.80 & 0.97 \\
South & 0.99 & 0.79 & 0.96 & 0.72 \\
East & 0.98 & 0.96 & 0.86 & 0.88 \\
North & 0.97 & 0.86 & 0.96 & 0.70 \\
\hline
\end{tabular}

open boundary, suggesting the reasonability and feasibility of the model. In addition, the inverse results in TEla are better than those in TE1b, revealing the feasibility and efficiency of IP strategy. Combining all these results, it can be concluded that the $3 \mathrm{D}$ internal tidal model involving adjoint method is suitable to invert OBCs and the IP strategy is useful in the estimation of OBCs.

3.2. Inversion by Assimilating "Inaccurate Observations". Considering that practice in situ observations may contain noises, artificial random errors are added to "observations." In this section, based on the "observations" in TEla, five TEs are carried out and the maximum percentages of error are $10 \%, 20 \%, 30 \%, 40 \%$, and $50 \%$ in TE2a, TE2b, Te2c, TE2d and TE2e, respectively. Then these "observations" are assimilated to invert OBCs. Results are displayed in Figures 5, 6, and 7.
Figure 5 illustrates the inverse results. All the shapes of inverse $\mathrm{OBC}$ are consistent with those of the prescribed ones. It is found that with increased artificial errors, the inverted OBCs become further away from the prescribed values, implying that larger errors will result in worse inversion of OBCs. The cost function and its gradient norm are plotted in Figure 6. After assimilation, the cost function is about $1 \%$ of its initial value in TEla and about 3\% in TE2e. Combined with MAEs and RCs between inverted OBCs and prescribed ones in Figure 7, where all MAEs are less than $3.5 \mathrm{~cm}$ and RCs are larger than 0.6, it is reasonable to believe that the prescribed OBCs have been inverted successfully though assimilating the "observations" is not accurate. But it cannot be denied that as artificial errors increased, the inversion results become worse with larger MAEs and smaller RCs. In addition, in these TEs, the inversion results of $a$ are better than those of $b$.

\section{Practical Experiments}

The 3D internal tidal model is used to simulate the $M_{2}$ internal tide around Hawaii by assimilating T/P data with OBCs optimized. IP strategy is used, which is the same as that used in TEla. Both locations and values of observations are used in PE, which is different from TEs. In PE, the iterative assimilation terminates once the number of iteration steps reaches 50 exactly.

After assimilation, the cost function is about $3 \%$ of its initial value. The simulated surface cochart is illustrated in Figure 8. Considering the co-phase lines are disordered due to the influence of internal tide, only coamplitude lines are contoured. On the whole, the simulated cochart shows good agreement with that obtained from Oregon State University (OSU) tidal model in which only barotropic tide is simulated. The sea surface amplitude is about $25 \mathrm{~cm}$ at the southeast of Hawaii and smaller than $15 \mathrm{~cm}$ at the northwest. Careful inspection shows that the major difference between simulated results and OSU model results is in the short-scale fluctuations superposed on co-amplitude lines. These fluctuations can be considered as the surface manifestation modulated by the internal tide, from which the propagation of internal tide could be found. The $M_{2}$ internal tide generated along the Hawaiian Ridge mainly propagates towards to northward and southeastward.

Figure 9 illustrates the difference of surface amplitude along tracks $006,045,082,147,184$, and 223. Simulated results show good agreement with observations. Table 2 shows the corresponding MAEs. All MAEs of amplitude and phase are less than $2 \mathrm{~cm}$ and $8^{\circ}$ (Table 3 ), respectively. Relative errors of amplitude are about 0.1, smaller than those in Ray and Mitchum [3], suggesting the advantage of this 3D internal tidal model involving adjoint method in simulating internal tides.

Compared with the barotropic results from OSU tidal model, we can calculate the surface manifestation modulated by the internal tide, the maximum of which are 4.0, 4.2, 5.6, $3.6,9.1$, and $6.7 \mathrm{~cm}$, corresponding to tracks 006, 045, 082, 147, 184 , and 223, respectively. Except the manifestation of $9.1 \mathrm{~cm}$ 
TABLE 3: MAEs of amplitude and phase between observations and simulated results (values in brackets are mean relative errors of amplitude).

\begin{tabular}{lcccccc}
\hline & 006 & 045 & 082 & 147 & 182 & 223 \\
\hline Amplitude $(\mathrm{cm})$ & $1.0(0.07)$ & $0.8(0.04)$ & $1.1(0.06)$ & $1.4(0.11)$ & $1.8(0.14)$ & $1.6(0.10)$ \\
Phase $\left(^{\circ}\right)$ & 4.3 & 3.0 & 4.7 & 6.0 & 7.7 & 5.9 \\
\hline
\end{tabular}

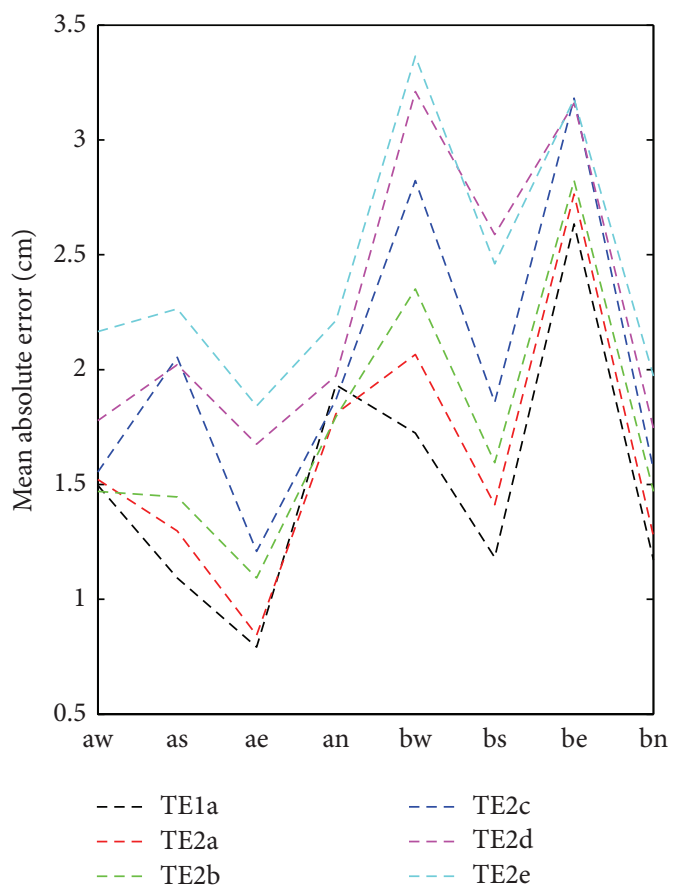

(a)

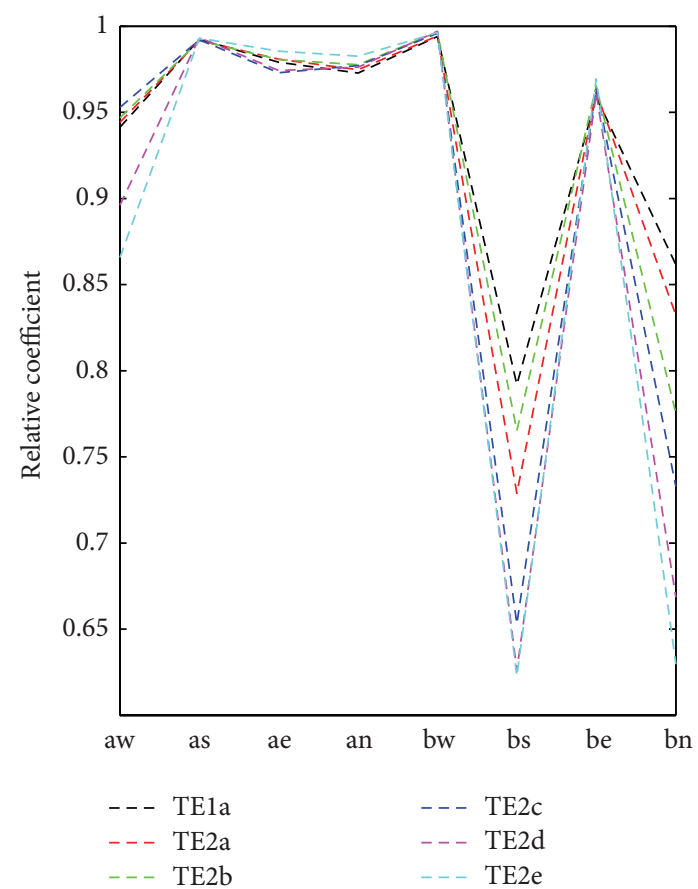

(b)

Figure 7: MAEs and RCs in TE2 (aw, bw, as, bs, ae, be, an, and bn represent Fourier coefficients on west, south, east, and north open boundaries, respectively).

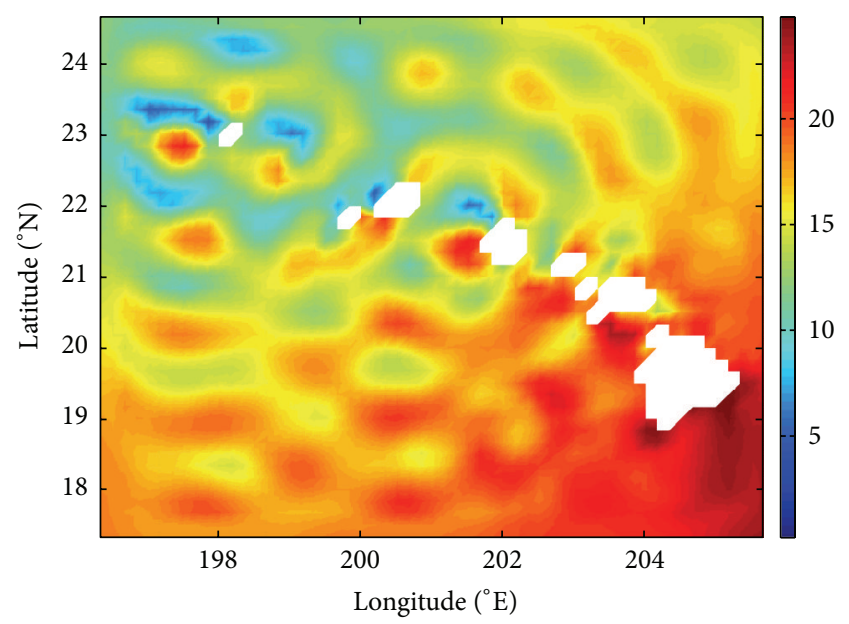

(a)

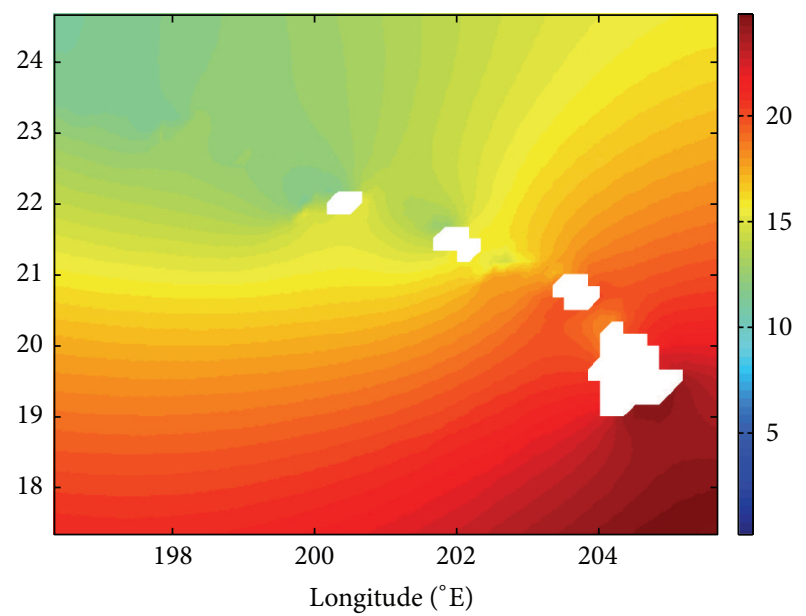

(b)

FIGURE 8: Simulated co-chart (unit: $\mathrm{cm},(\mathrm{a})$ ) and that from OSU tidal model (b).

along track 184, the values along other tracks are consistent with the estimation of Ray and Mitchum [3, 4].

\section{Conclusion and Summary}

In this paper, the optimization of OBCs in a 3D internal tidal model is investigated with the adjoint method. Fourier coefficients of $M_{2}$ internal tide on four open boundaries are inverted simultaneously, which means more variables are estimated in the inverse problem. Special step factor is used in the steepest descent method in order to minimize cost function efficiently without fluctuations. The reasonability and feasibility of the model and IP strategy are tested by TE1. In 

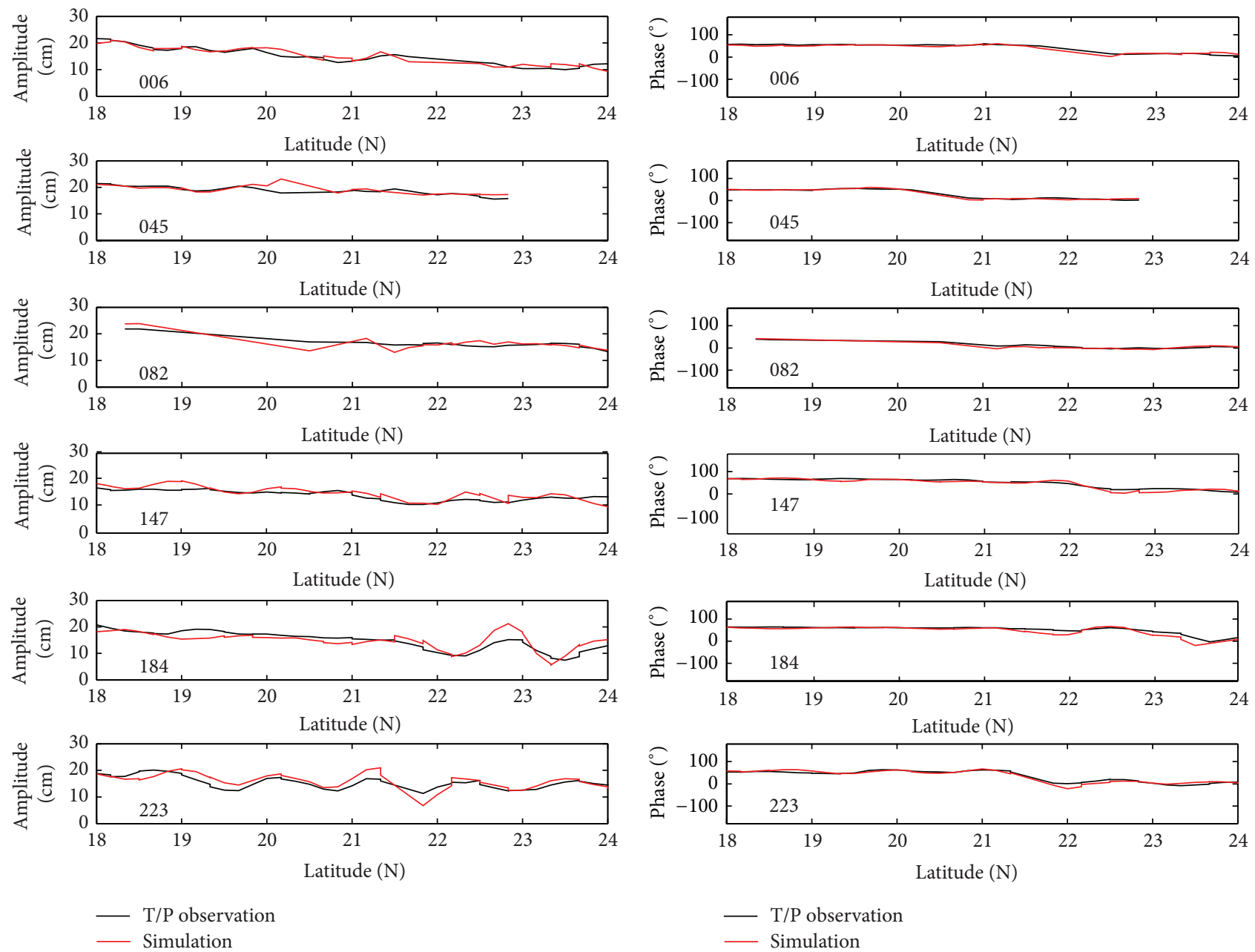

(a)

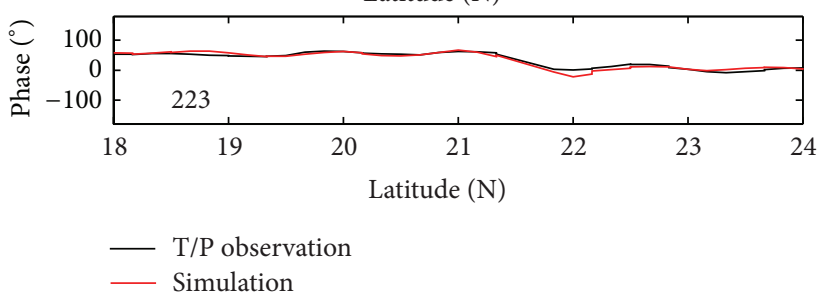

(b)

FIGURE 9: Observations and simulated results along tracks 006, 045, 082, 147, 184, and 223.

TE2, artificial random errors, of which the percentage is from $10 \%$ to $50 \%$, are added to observations. After assimilation, the cost function reaches less than 3\% of its initial values MAEs and RCs between inverted OBCs and prescribed ones are less than $3.5 \mathrm{~cm}$ and larger than 0.6 , respectively, indicating that the prescribed OBCs are inverted successfully. But it cannot be denied that as artificial errors increased, the inversion results become worse gradually.

In $\mathrm{PE}$, the OBCs are optimized and the $M_{2}$ internal tide around Hawaii is simulated by assimilating T/P data. The simulated co-chart shows good agreement with that from Oregon State University (OSU) tidal model and T/P observations on the whole. Careful inspection shows that the major difference between simulated results and OSU model results is in the short-scale fluctuations superposed on co-amplitude lines, which can be treated as the surface manifestation modulated by the internal tide. The maximum manifestation along tracks 006, 045, 082, 147, 184, and 223 are $4.0,4.2,5.6,3.6,9.1$, and $6.7 \mathrm{~cm}$, receptively. Except the manifestation of $9.1 \mathrm{~cm}$ along track 184, the values along other tracks are consistent with the estimation of Ray and Mitchum $[3,4]$.

\section{Acknowledgments}

Partial support for this research was provided by the National Natural Science Foundation of China through Grants 41076006 and 41206001, Natural Science Foundation of Jiangsu Province of China through Grant BK2012315, the State Ministry of Science and Technology of China through Grants 2013AA091201 and 2013AA091202, and the Fundamental Research Funds for the Central Universities 201261006 and 201262007.

\section{References}

[1] D. L. Rudnick, T. J. Boyd, R. E. Brainard et al., "From tides to mixing along the Hawaiian Ridge," Science, vol. 301, no. 5631, pp. 355-357, 2003. 
[2] W. Munk and C. Wunsch, "Abyssal recipes II: energetics of tidal and wind mixing," Deep-Sea Research Part I, vol. 45, no. 12, pp. 1977-2010, 1998.

[3] R. D. Ray and G. T. Mitchum, "Surface manifestation of internal tides generated near Hawaii," Geophysical Research Letters, vol. 23, no. 16, pp. 2101-2104, 1996.

[4] R. D. Ray and G. T. Mitchum, "Surface manifestation of internal tides in the deep ocean: observations from altimetry and island gauges," Progress in Oceanography, vol. 40, no. 1-4, pp. 135-162, 1997.

[5] W. Munk, "Once again: once again-tidal friction," Progress in Oceanography, vol. 40, no. 1-4, pp. 7-35, 1997.

[6] J. P. Martin, D. L. Rudnick, and R. Pinkel, "Spatially broad observations of internal waves in the upper ocean at the Hawaiian Ridge," Journal of Physical Oceanography, vol. 36, no. 6, pp. 1085-1103, 2006.

[7] Z. Zhao, M. H. Alford, J. A. Mackinnon, and R. Pinkel, "Longrange propagation of the semidiurnal internal tide from the Hawaiian Ridge," Journal of Physical Oceanography, vol. 40, no. 4, pp. 713-736, 2010.

[8] Z. Zhao, M. Alford, J. Girton, T. Johnston, and G. Carter, "Internal tides around the Hawaiian Ridge estimated from multisatellite altimetry," Journal of Geophysical Research, vol. 116, Article ID C12039, 2011.

[9] Z. Zhao, M. Alford, and J. Griton, "Mapping low-mode internal tides from mulsatellite altimetry," Oceanography, vol. 25, no. 2, pp. 42-51, 2012.

[10] S. K. Kang, M. G. G. Foreman, W. R. Crawford, and J. Y. Cherniawsky, "Numerical modeling of internal tide generation along the Hawaiian Ridge," Journal of Physical Oceanography, vol. 30, no. 5, pp. 1083-1098, 2000.

[11] H. Simmons, R. Hallberg, and B. Arbic, "Internal wave generation in a global baroclinic tide model," Deep-Sea Research II, vol. 51, pp. 3043-3068, 2004.

[12] G. S. Carter, M. A. Merrifield, J. M. Becker et al., "Energetics of $\mathrm{M}_{2}$ barotropic-to-baroclinic tidal conversion at the Hawaiian Islands," Journal of Physical Oceanography, vol. 38, no. 10, pp. 2205-2223, 2008.

[13] B. Powell, I. Janekovic, G. Carter, and M. A. Merrifield, "Sensitivity of internal tide generation in Hawaii," Geophysical Research Letters, vol. 39, Article ID L10606, 2012.

[14] L. Rainville, T. M. S. Johnston, G. S. Carter et al., "Interference pattern and propagation of the $\mathrm{M}_{2}$ internal tide south of the Hawaiian Ridge," Journal of Physical Oceanography, vol. 40, no. 2, pp. 311-325, 2010.

[15] E. D. Zaron and G. D. Egbert, "The impact of the $M_{2}$ internal tide on data-assimilative model estimates of the surface tide," Ocean Modelling, vol. 18, no. 3-4, pp. 210-216, 2007.

[16] I. Shulman, "Local data assimilation in specification of open boundary conditions," Journal of Atmospheric and Oceanic Technology, vol. 14, no. 6, pp. 1409-1419, 1997.

[17] I. Shulman, J. K. Lewis, A. F. Blumberg, and B. N. Kim, "Optimized boundary conditions and data assimilation with application to the $\mathrm{M}_{2}$ tide in the yellow sea," Journal of Atmospheric and Oceanic Technology, vol. 15, no. 4, pp. 10661071, 1998.

[18] V. Taillandier, V. Echevin, L. Mortier, and J. L. Devenon, "Controlling boundary conditions with a four-dimensional variational data-assimilation method in a non-stratified open coastal model," Ocean Dynamics, vol. 54, no. 2, pp. 284-298, 2004.
[19] P. G. J. ten Brummelhuis, A. W. Heemink, and H. F. P. van den Boogaard, "Identification of shallow sea models," International Journal for Numerical Methods in Fluids, vol. 17, no. 8, pp. 637665, 1993.

[20] E. Kazantsev, "Boundary conditions control for a shallow-water model," International Journal for Numerical Methods in Fluids, vol. 68, no. 5, pp. 625-641, 2012.

[21] I. Yu. Gejadze and G. J. M. Copeland, "Open boundary control problem for Navier-Stokes equations including a free surface: adjoint sensitivity analysis," Computers \& Mathematics with Applications, vol. 52, no. 8-9, pp. 1243-1268, 2006.

[22] R. Lardner, "Optimal assimilation of current and surface elevation data in a two-dimensional numerical tidal model," Applied Mathematical Modelling, vol. 19, pp. 30-38, 1995.

[23] E. P. Myers and A. M. Baptista, "Inversion for tides in the Eastern North Pacific Ocean," Advances in Water Resources, vol. 24, no. 5, pp. 505-519, 2001.

[24] J. Zhang and X. Lu, "Inversion of three-dimensional tidal currents in marginal seas by assimilating satellite altimetry," Computer Methods in Applied Mechanics and Engineering, vol. 199, no. 49-52, pp. 3125-3136, 2010.

[25] Z. Guo, A. Cao, and X. Lv, "Inverse estimation of open boundary conditions in the Bohai Sea," Mathematical Problems in Engineering, vol. 2012, Article ID 628061, 9 pages, 2012.

[26] A. Cao, Z. Guo, and X. Lv, "Inversion of two-dimensional tidal open boundary conditions of $\mathrm{M}_{2}$ constituent in the Bohai and Yellow Seas," Chinese Journal of Oceanology and Limnology, vol. 30, no. 5, pp. 868-875, 2012.

[27] H. Chen, C. Miao, and X. Lv, "A three-dimensional numerical internal tidal model involving adjoint method," International Journal for Numerical Methods in Fluids, vol. 69, no. 10, pp. 15841613, 2012.

[28] X. Lu and J. Zhang, "Numerical study on spatially varying bottom friction coefficient of a 2D tidal model with adjoint method," Continental Shelf Research, vol. 26, no. 16, pp. 19051923, 2006.

[29] J. Zhang, X. Lu, P. Wang, and Y. P. Wang, "Study on linear and nonlinear bottom friction parameterizations for regional tidal models using data assimilation," Continental Shelf Research, vol. 31, no. 6, pp. 555-573, 2011.

[30] L. Yu and J. O'Brien, "Variational estimation of the wind stress drag coefficient and the oceanic eddy viscosity profile," Journal of Physical Oceanography, vol. 21, pp. 709-719, 1990.

[31] C. Miao, H. Chen, and X. Lv, "An isopycnic-coordinate internal tide model and its application to the South China Sea," Chinese Journal of Oceanology and Limnology, vol. 29, no. 6, pp. 13391356, 2011.

[32] M. Gunzburger, "Adjoint equation-based methods for control problems in incompressible, viscous flows," Flow, Turbulence and Combustion, vol. 65, no. 3-4, pp. 249-272, 2000.

[33] G. I. Marchuk, "Perturbation theory and the statement of inverse problems," in Fifth Conference on Optimization Techniques (Rome, 1973), vol. 4 of Lecture Notes in Comput. Sci., pp. 159-166, Springer, Berlin, Germany, 1973.

[34] G. Marchuk, "Basic and adjoint equations of atmosphere and ocean dynamics," MeteorologIya i GIdrologIya, vol. 2, no. 9, pp. 9-37, 1974.

[35] D. G. Cacuci, "Sensitivity theory for nonlinear systems. II. Extensions to additional classes of responses," Journal of Mathematical Physics, vol. 22, no. 12, pp. 2803-2812, 1981. 
[36] G. I. Marchuk, Adjoint Equations and Analysis of Complex Systems, vol. 295 of Mathematics and Its Applications, Kluwer Academic Publishers, Dordrecht, The Netherlands, 1995.

[37] I. M. Navon, "Practical and theoretical aspects of adjoint parameter estimation and identifiability in meteorology and oceanography," Dynamics of Atmospheres and Oceans, vol. 27, no. $1-4$, pp. 55-79, 1998.

[38] R. A. Flather, "A tidal model of the north-west European continental shelf," Memoires de la Societe Royale des Sciences de Liege, vol. 6, no. 10, pp. 141-164, 1975. 


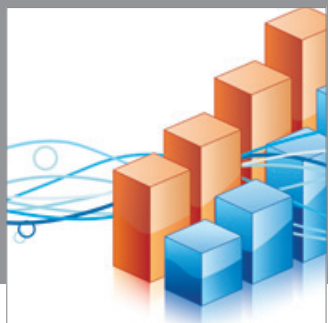

Advances in

Operations Research

mansans

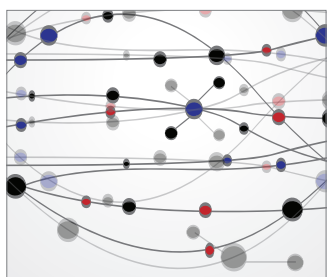

The Scientific World Journal
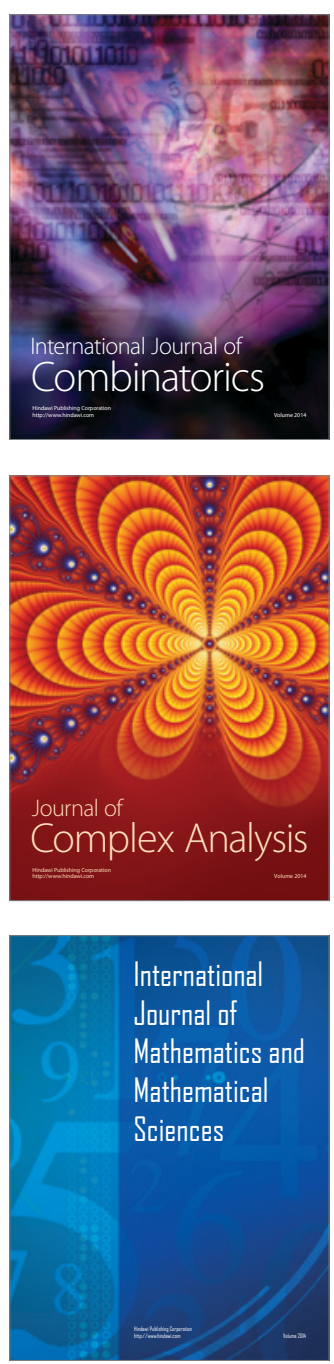
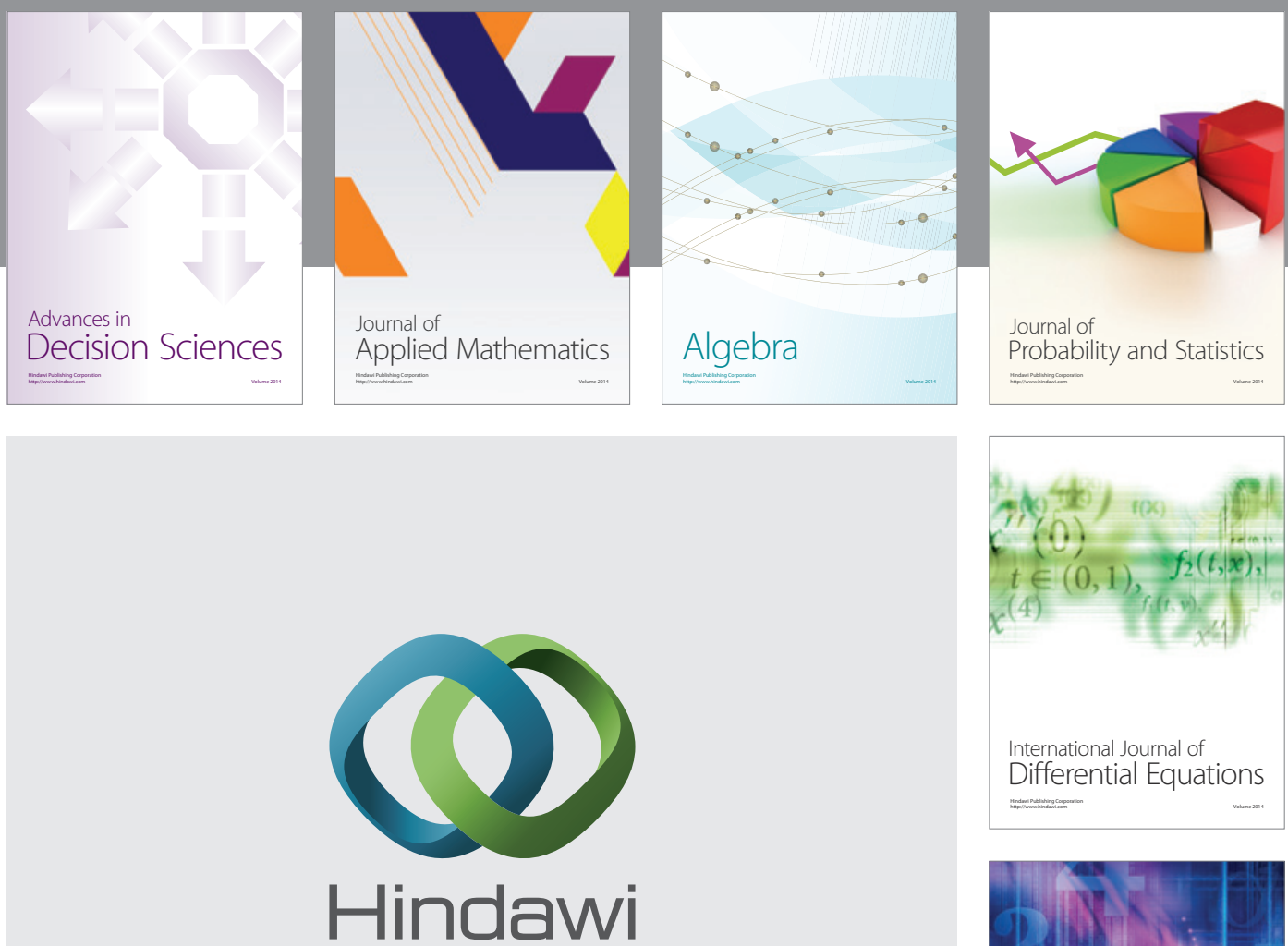

Submit your manuscripts at http://www.hindawi.com
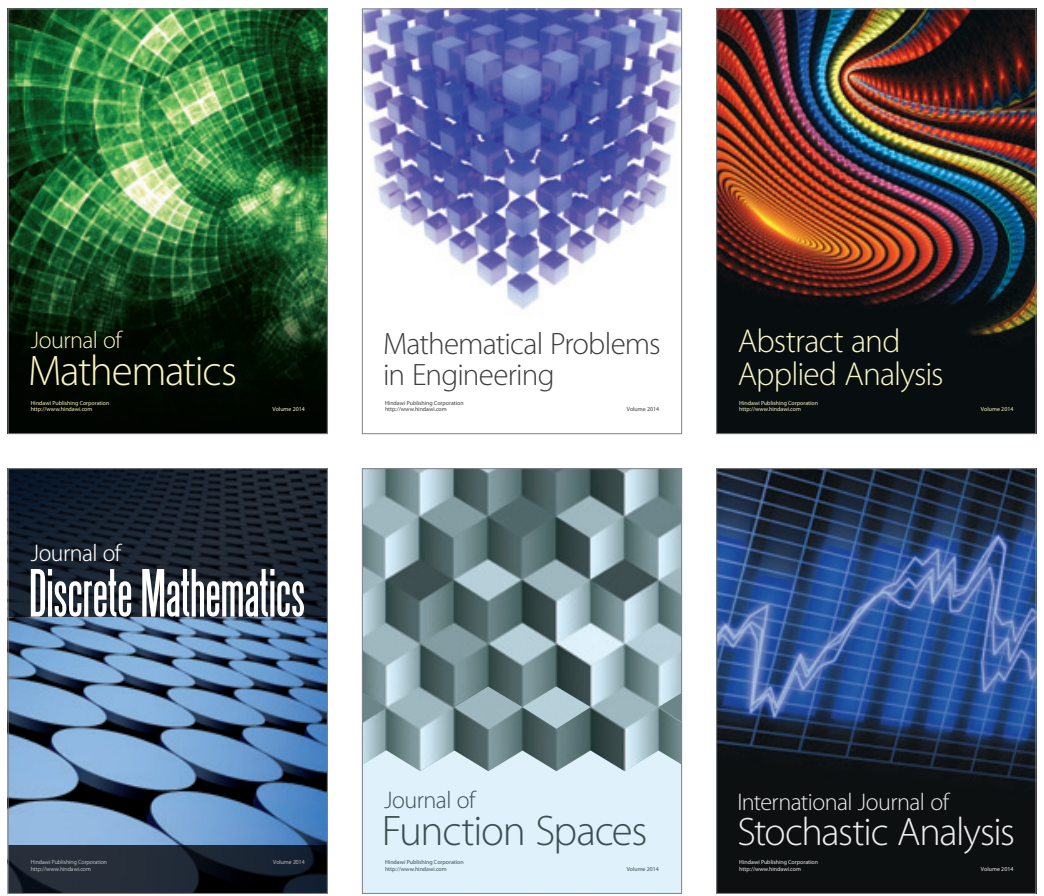

Journal of

Function Spaces

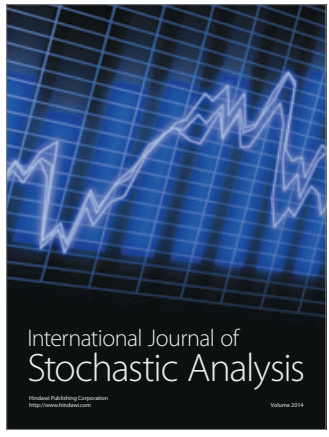

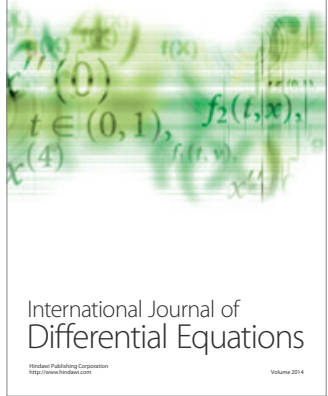
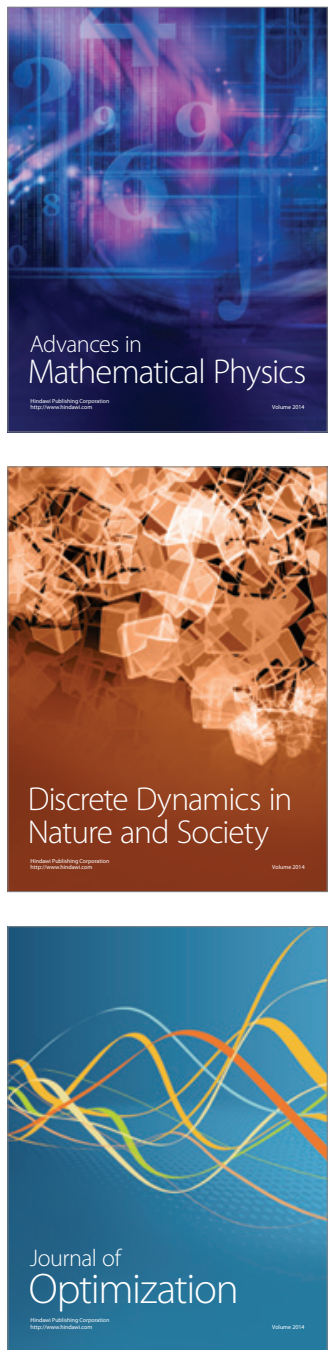